\title{
Synergistic inhibition effect of TNIK inhibitor KY-05009 and receptor tyrosine kinase inhibitor dovitinib on IL-6-induced proliferation and Wnt signaling pathway in human multiple myeloma cells
}

\author{
Yura Lee ${ }^{1,4}$, Jung-Il Jung ${ }^{1}$, Kyeong-Yong Park ${ }^{2}$, Soon Ae Kim ${ }^{3}$ and Jiyeon Kim ${ }^{1}$ \\ ${ }^{1}$ Department of Biomedical Laboratory Science, School of Medicine, Eulji University, Daejeon 34824, Korea \\ ${ }^{2}$ R\&D Center, Peptron, Inc., Daejeon 34054, Korea \\ ${ }^{3}$ Department of Pharmacology, School of Medicine, Eulji University, Daejeon 34824, Korea \\ ${ }^{4}$ Present address: Severance Biomedical Science Institute, Brain Korea 21 PLUS Project for Medical Science, College of \\ Medicine, Yonsei University, Seoul 03722, Korea \\ Correspondence to: Jiyeon Kim, email: yeon@eulji.ac.kr \\ Keywords: multiple myeloma, TNIK, KY-05009, IL-6, Wnt signaling \\ Received: December 06, $2016 \quad$ Accepted: March 21, $2017 \quad$ Published: April 12, 2017 \\ Copyright: Lee et al. This is an open-access article distributed under the terms of the Creative Commons Attribution License 3.0 \\ (CC BY 3.0), which permits unrestricted use, distribution, and reproduction in any medium, provided the original author and source \\ are credited.
}

\section{ABSTRACT}

Multiple myeloma is a fetal form of plasma cell malignancy characterized by abnormal clonal proliferation of plasma cells. Especially, the canonical Wnt signaling pathway mediated by $\beta$-catenin is activated in multiple myeloma cells, stimulating their proliferation. Here, we investigated the relationship between interleukin6-induced proliferation of multiple myeloma cells and Traf2- and Nck-interacting kinase (TNIK) expression in Wnt signaling. Interleukin-6 increased the proliferation of multiple myeloma cells and TNIK mRNA and protein expression. In addition, we examined the effect on TNIK of TNIK inhibitor KY-05009 and receptor tyrosine kinase inhibitor dovitinib and whether inhibition of TNIK suppresses the interleukin-6induced proliferation of multiple myeloma cells. KY-05009 and dovitinib synergistically inhibited interleukin-6-stimulated proliferation and induced apoptosis through the inhibition of Wnt signaling in MM cells. Our results provide crucial information that TNIK is involved in the interleukin-6-dependent proliferation of multiple myeloma cells and inhibition of Wnt signaling involving TNIK could be a therapeutic strategy for the treatment of interleukin-6-dependent multiple myeloma.

\section{INTRODUCTION}

Multiple myeloma (MM) is a plasma cell malignancy characterized by excess clonal proliferation of abnormal plasma cells in the bone marrow, elevated secretion of monoclonal proteins in the serum or urine, and multiple organ damage, including renal failure, hypercalcemia, anemia, and lytic bone resorption [1]. $\mathrm{MM}$ is estimated to account for approximately $10 \%$ of all hematological malignancies but remains an incurable hematological disorder, despite advances in the introduction of new drugs and therapies [2]. New drugs, such as thalidomide, lenalidomide, and bortezomib, have been used for the treatment of relapsed and refractory
$\mathrm{MM}$, but the median survival has remained 5-6 years in the elderly [3]. To increase the survival rate in MM patients, new therapeutic strategies using novel drugs are needed.

The serine/threonine kinase Traf2- and Nckinteracting kinase (TNIK), a member of the germinal center kinase (GCK) family, was first identified as a regulatory kinase in cell spreading or migration through cytoskeleton organization $[4,5]$. In the last 10 years, TNIK has been reported as a novel therapeutic target in several types of cancers. In many studies, the expression of TNIK has been shown to be involved in the survival of human cancer cells, including colorectal, gastric, liver, and hematological cancer [6-11]. Wnt signaling is mediated by TNIK through interactions with $\beta$-catenin and following phosphorylation 
of T-cell factor (TCF) 4 at serine 154 in the nucleus. Phosphorylation of TCF4 induces the activation of Wnt target genes, such as CCND1, AXIN2, ZCCHC12, and TCF7 [6]. In addition, the inhibition of TNIK expression by small-interfering RNA (siRNA) suppresses the transcriptional activity of TCF/lymphoid enhancer-binding factor (LEF) and induces apoptosis [7, 11]. A few reports have demonstrated the expression of TNIK and cancer cell proliferation in several types of cancer, but the relevance of TNIK to hematological malignancies, especially MM, has not been sufficiently described [6-11].

In our previous studies, we investigated the apoptosis-inducing effect of tyrosine kinase inhibitor dovitinib and its inhibition of TNIK kinase activity and endogenous Wnt signaling in human MM cells [11]. TNIK is highly expressed in MM cells compared to normal peripheral blood mononuclear cells (PBMCs), and inhibition of TNIK expression by siRNA induces cell death. KY-05009 and dovitinib have a high affinity for the ATP binding site in TNIK and inhibit the protein expression of TNIK and transcriptional activity of Wnt target genes $[11,12]$. Through these our recent reports, we confirmed that TNIK can be a potential target for inducing apoptosis activity of KY-05009 and dovitinib in cancer cells.
In the present study, we investigated the level of TNIK expression in human MM cells from patients and the apoptosis-inducing effect of KY-05009 and dovitinib in the IL-6-dependent MM RPMI8226 cell line. IL-6 enhanced cell proliferation, TNIK mRNA and protein expression, and the transcriptional activity of Wnt target genes. KY-05009 exerted synergistic anti-proliferative effects with dovitinib and triggered caspase-dependent apoptosis in RPMI8226 cells. We hypothesize that a possible mode of action of KY-05009 and dovitinib is a high affinity for TNIK and subsequent inhibition of kinase activity $[11,12]$. This inhibitory effect against TNIK may suppress the proliferation of RPMI8226 cells. Thus, our results suggest that TNIK could be an anti-cancer target for the investigation of treating MM by inhibiting Wnt signaling-mediated MM cell proliferation.

\section{RESULTS}

\section{IL-6 stimulates the proliferation of RPMI8226 cells}

IL-6 has been identified as a major growth factor for myeloma cell proliferation in vivo and in vitro [13-16]. In particular, paracrine regulation of IL-6
A
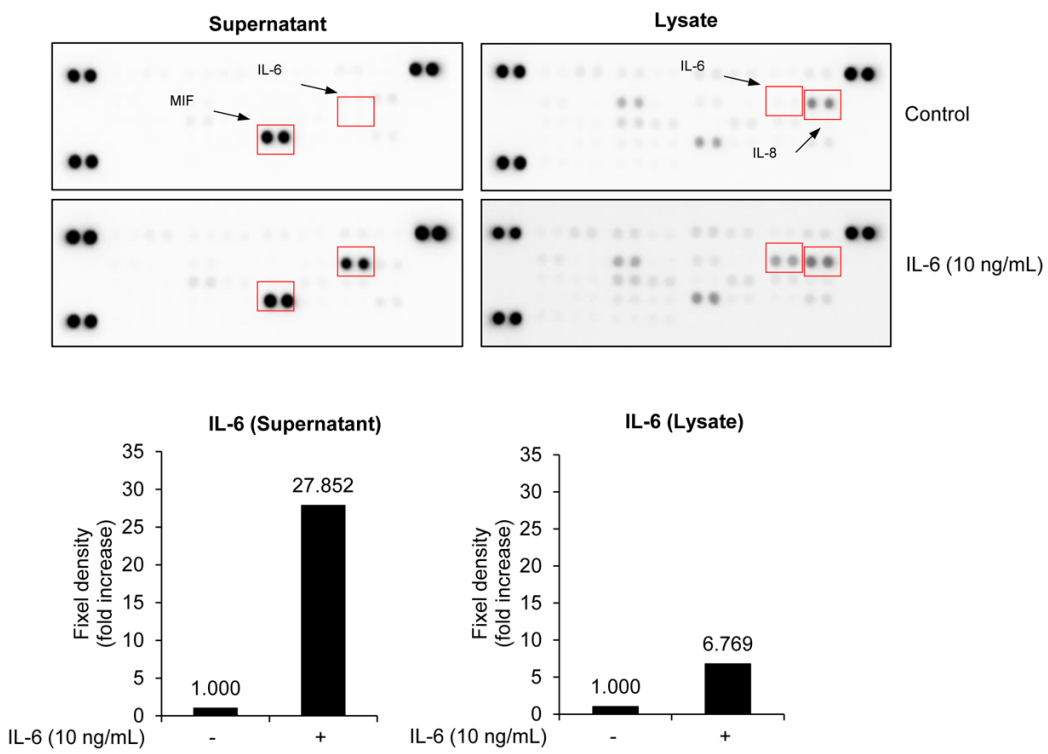

B
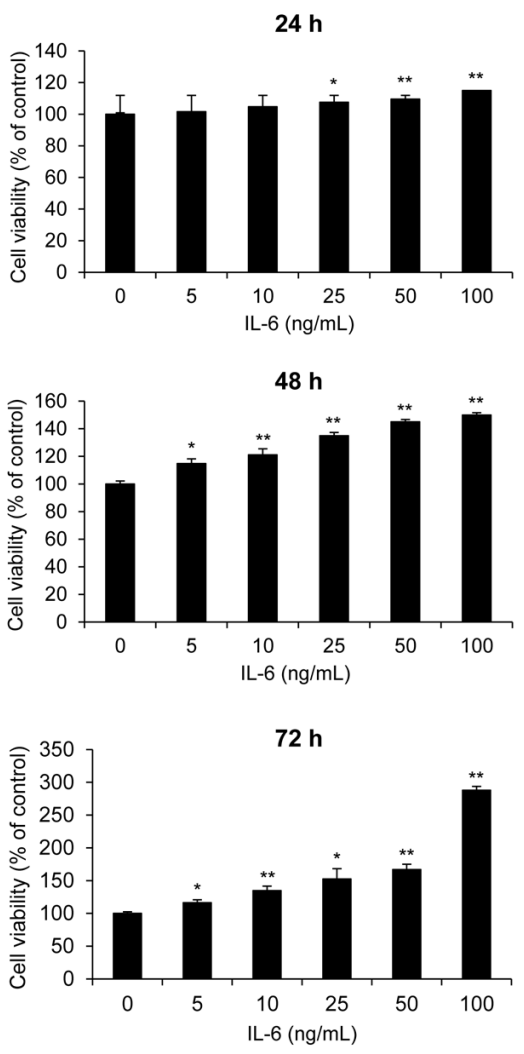

Figure 1: IL-6 activates MM cell proliferation. (A) RPMI8226 cells were treated with recombinant human IL-6 for $72 \mathrm{~h}$. After incubation, cytokine expression in cell supernatants and lysates was analyzed by human cytokine array. The expression of IL-6 was normalized by the density of control spots. (B) Cell viability of RPMI8226 cells after treatment with IL-6 (0-100 ng/mL) in serum-free medium for 24-72 h. Data are presented as mean \pm SD. The experiments were performed in triplicate. ${ }^{*} P<0.01,{ }^{* *} P<0.001$ versus control. 
stimulates myeloma cell proliferation in patients [13]. To confirm the effect of IL-6 on the production of cytokines in MM cells, we analyzed the expression of cytokines and whether IL-6 treatment induces paracrine effects of other cytokines, such as IL-1, IL-2, IL-4, IL-5, and tumor necrosis factor (TNF)- $\alpha$, on cultured supernatant or protein expression in cell lysates (Figure 1A). Serum-starved RPMI8226 cells were treated with recombinant human IL-6 in serum-free medium for $72 \mathrm{~h}$ and the culture supernatants and cell lysates isolated to analyze secreted factors and their influence on protein expression in MM cells. A human cytokine array showed that migration inhibitor factor (MIF), an inflammatory mediator, was constitutively produced regardless of IL- 6 treatment. IL- 6 was only expressed in the supernatant in response to IL-6 treatment (Figure 1A, left). We also observed that IL-8, an activator of osteoclast differentiation and bone resorption in MM, was expressed in both untreated controls and the IL-6treated group, but IL- 6 only increased in the IL-6-treated lysate group (Figure 1A, right). Next, we assessed the stimulatory effect of IL-6 on the proliferation of MM cells. RPMI8226 cells were incubated with recombinant human IL-6 for 24 to $72 \mathrm{~h}$. As shown in Figure 1B, IL-6 stimulated the proliferation of MM cells in a doseand time-dependent manner. These results support an increased level of IL-6 in cultured supernatants and cell lysates correlating with MM cell proliferation.

\section{IL-6 activates TNIK expression and the transcriptional activity of Wnt signaling}

Our previous studies demonstrated the association between canonical Wnt signaling and the survival of $\mathrm{MM}$ cells [17, 18]. In addition, targeting of the constitutively active $\beta$-catenin/TCF4 transcriptional complex has been identified as a potent therapeutic strategy in the treatment of MM [19]. Several reports have shown that TNIK activates the transcription of Wnt target genes through interactions with $\beta$-catenin and TCF4 and following the phosphorylation of TCF4 at serine $154[6,7,11]$. In this study, we confirmed the IL-6-induced transcriptional activity of Wnt target genes and TNIK protein expression using RPMI8226 cells. First, we determined TNIK mRNA expression in human MM patients $(n=15)$ (Supplementary Figure 1). The relative endogenous TNIK mRNA expression was increased compared to primary PBMCs. To evaluate the relationship between IL-6 and the activation of TNIK expression and Wnt signaling, we detected the IL-6induced transcriptional activity of TNIK and Wnt target genes. As shown in Figure 2A and Supplementary Figure 2, IL-6 treatment activated TNIK mRNA expression and Wnt signaling-related genes, including CTNNB1, TCF4, and TCF7 until 60 minutes (Table 1).

IL-6 treatment induced translocation of cytosolic TNIK into the nucleus in a time-dependent manner (Figure
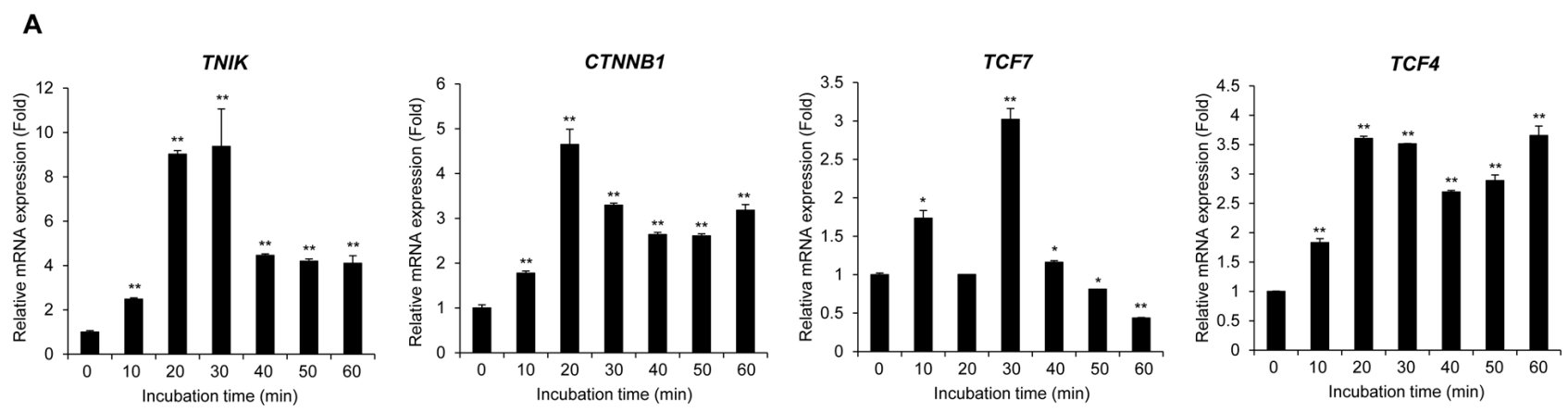

B

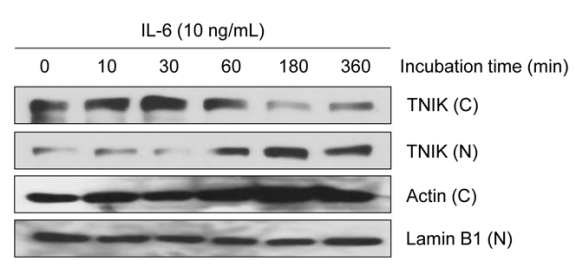

C

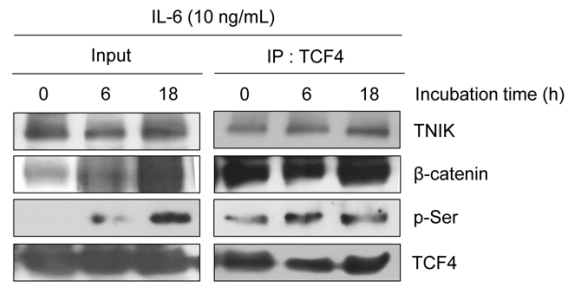

Figure 2: IL-6 activates TNIK expression and Wnt target gene transcription. (A) qRT-PCR analysis of the indicated genes in serum-starved RPMI8226 cells after treatment with IL-6 $(10 \mathrm{ng} / \mathrm{mL})$ for 0-30 min. Data are presented as mean \pm SD. Experiments were performed in triplicate. ${ }^{*} P<0.01,{ }^{* *} P<0.001$ versus control. (B) Western blot of TNIK expression in the cytosolic (C) and nuclear (N) fractions of RPMI8226 cells treated with IL-6 (10 ng/mL) in serum-free medium for $0-360 \mathrm{~min}$. Actin was used as a loading control. (C) Phosphorylation of TCF4 and expression of TCF4-bound proteins were measured by immunoprecipitation and western blot analysis of RPMI8226 cells treated with IL-6 (10 ng/mL) in serum-free medium for 0, 6, and $18 \mathrm{~h}$. 
Table 1: Primer sequences used in this study

\begin{tabular}{lll}
\hline Target gene & Forward $\left(\mathbf{5}^{\prime} \mathbf{-} \mathbf{3}^{\prime}\right)$ & Reverse $\left(\mathbf{5}^{\prime} \mathbf{- 3} \mathbf{3}^{\prime}\right)$ \\
\hline$T C F 7$ & CTGCACATGCAGCTATACCC & GGCCACCTGTCTCTGAGATT \\
$C T N N B 1$ & CCATCTTCCAGGAGCGAGAT & CAGTGATGGCATGGACTGTG \\
$T C F 4$ & GAGCAGCAAGTCCGAGAAAG & ATGCTGAAACCTCTTGCGTC \\
$T N I K$ & GCTATTGAGATCCGGTCAGT & CAGGCTGCAACATTGAAAGA \\
GAPDH & GAGTCAACGGATTTGGTCGT & GATCTCGCTCCTGGAAGATG \\
\hline
\end{tabular}

2B). We also used co-immunoprecipitation to confirm the effect of IL-6 on the activation of Wnt signaling. As shown in Figure 2C, IL-6 treatment induced the phosphorylation of TCF4. These results indicate that IL-6-induced MM cell proliferation is related to the expression of TNIK and activation of the Wnt signaling pathway in MM cells.

\section{Inhibition of TNIK induces caspase-dependent apoptosis in MM cells}

In a previous study, we found that the inhibition of TNIK by dovitinib or siRNA suppresses the expression of endogenous Wnt signaling regulatory proteins and MM survival [11]. We also confirmed that a novel aminothiazole TNIK inhibitor, KY-05009 (5-(4-methylbenzamido)-2(phenylamino) thiazole-4-carboxamide), has high affinity for the ATP binding site of TNIK and pharmacologically inhibits transforming growth factor (TGF)- $\beta 1$-induced epithelial-to-mesenchymal transition (EMT) in human lung adenocarcinoma cells [12]. In MM cells, dovitinib exerts anti-cancer activity through inhibition of TNIK kinase activity and the endogenous Wnt signaling pathway [11]. To confirm the association between TNIK inhibition and IL-6induced MM cell proliferation, we assessed the viability of RPMI8226 cells using KY-05009 (Figure 3A) and dovitinib (Figure 3B). As shown in Figure 3C and 3D, KY-05009 and dovitinib inhibited the proliferation of RPMI8226 cells, but there was also significant cytotoxicity in normal PBMCs.

Next, to determine the cell death-inducing mechanism of KY-05009 and dovitinib, we used flow cytometry to evaluate apoptosis. KY-05009 and dovitinib induced the binding of fluorescent Annexin $\mathrm{V}$ and 7-amino-actinomycin D (7-AAD) uptake, key features of late apoptosis, in a dose-dependent manner (Figure 4A) [20-22]. KY-05009 and dovitinib induced apoptosis at concentrations over $1 \mu \mathrm{M}$ (Figure $4 \mathrm{~B}$ and $4 \mathrm{C}$ ). This result suggests that inhibition of TNIK by kinase inhibitors could contribute to the induction of apoptosis of MM cells.

\section{KY-05009/dovitinib combination treatment synergistically induces apoptosis of MM cells}

We assessed cell viability and apoptosis in the presence of TNIK inhibition to determine whether KY-05009 and dovitinib cause synergistic effects in RPMI8226 cells (Figure 5A). The combination index (CI) was also calculated
(Table 2). Compared to the single treatment results, combined treatment had a synergistic effect. To evaluate whether this effect was due to apoptosis, we analyzed the population of early and late apoptotic cells using flow cytometry. As shown in Figure 5B, combined treatment had a synergistic apoptosis-inducing effect compared to the single compound treatment in Figure 4A. Furthermore, combined treatment increased the caspase-3/7 activity (Figure 5C) and cleavage of downstream apoptosis-related substrate poly (ADP-ribose) polymerase 1 (PARP-1) in the nucleus (Figure 5D). These results suggest that inhibition of TNIK using specific TNIK inhibitor KY-05009 and tyrosine kinase inhibitor dovitinib could have a synergistic effect on the induction of apoptosis of MM cells.

\section{KY-05009/dovitinib combination treatment synergistically inhibits IL-6-induced proliferation and activation of Wnt signaling}

To confirm the synergistic inhibitory effect of KY05009 and dovitinib on IL-6-induced activation of Wnt signaling in MM cells, we determined cell viability and performed a TOP/FOPflash luciferase assay. RPMI8226 cells were incubated with IL-6 alone or KY-05009 and/ or dovitinib for 24 or $48 \mathrm{~h}$. As shown in Figure 6A, IL-6 alone increased the cell proliferation rate, but KY-05009, dovitinib, and the combination of both decreased cell viability in a dose-dependent manner. The inhibitory effect of this combination treatment on cell proliferation was confirmed using other multiple myeloma cell lines such as IM-9 (IL-6-independent) and MM.1R (IL-6-dependent) cells (Supplementary Figure 3 and Supplementary Figure 4, Supplementary Table 1 and Supplementary Table 2). We also assessed the transcriptional activity of TCF4 using TOP/FOPflash reporter luciferase assay to determine whether inhibition of TNIK using KY-05009 and dovitinib suppresses the IL-6-induced activation of Wnt signaling. As shown in Figure 6B, KY-05009 and dovitinib inhibited the IL-6-induced TCF4-mediated transcription at $3 \mu \mathrm{M}$, and combination treatment significantly inhibited TCF4mediated transcriptional activity. We also examined the TNIK protein expression and transcriptional activity of Wnt target genes described in a previous report $[6,7,11]$. IL-6 increased the mRNA expression of Wnt signalingrelated genes, including TNIK, CTNNB1, TCF7, and 
A<smiles>Cc1ccc(C(=O)Nc2sc(Nc3ccccc3)nc2C(N)=O)cc1</smiles>

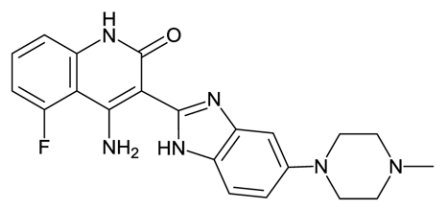

C
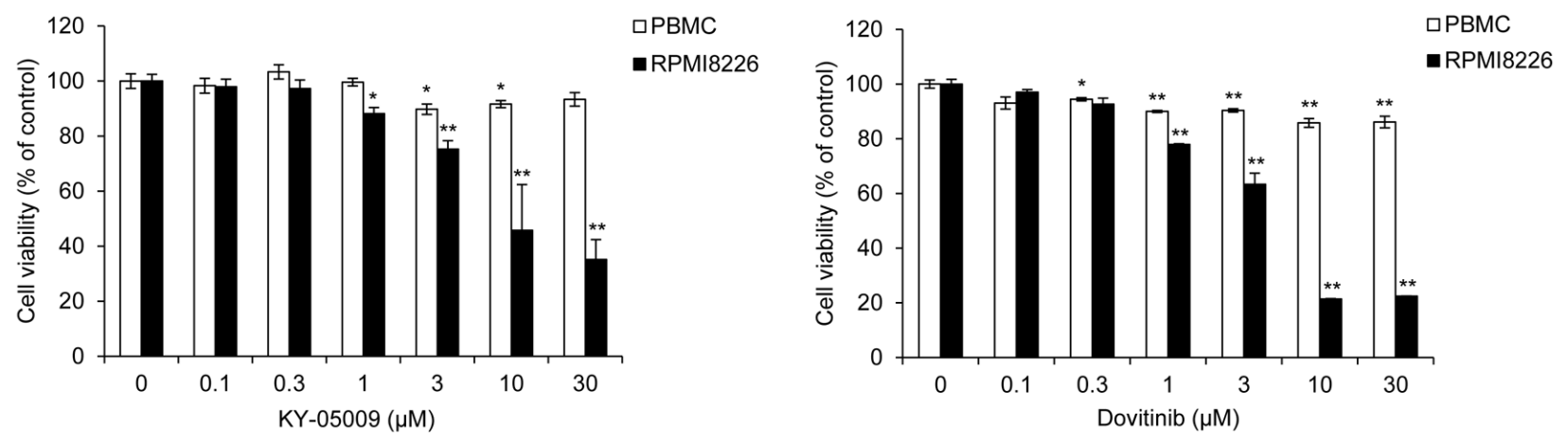

Figure 3: KY-05009 and dovitinib inhibit MM cell proliferation. (A) Chemical structure of KY-05009. (B) Chemical structure of dovitinib. (C and D) Cell viability of peripheral blood mononuclear cells (PBMCs) and RPMI8226 cells treated with KY-05009 (C) or dovitinib (D) in RPMI1640 medium containing 5\% FBS for $24 \mathrm{~h}$. Data are presented as mean \pm SD. Experiments were performed in triplicate. ${ }^{*} P<0.01,{ }^{* *} P<0.001$ versus control.

A

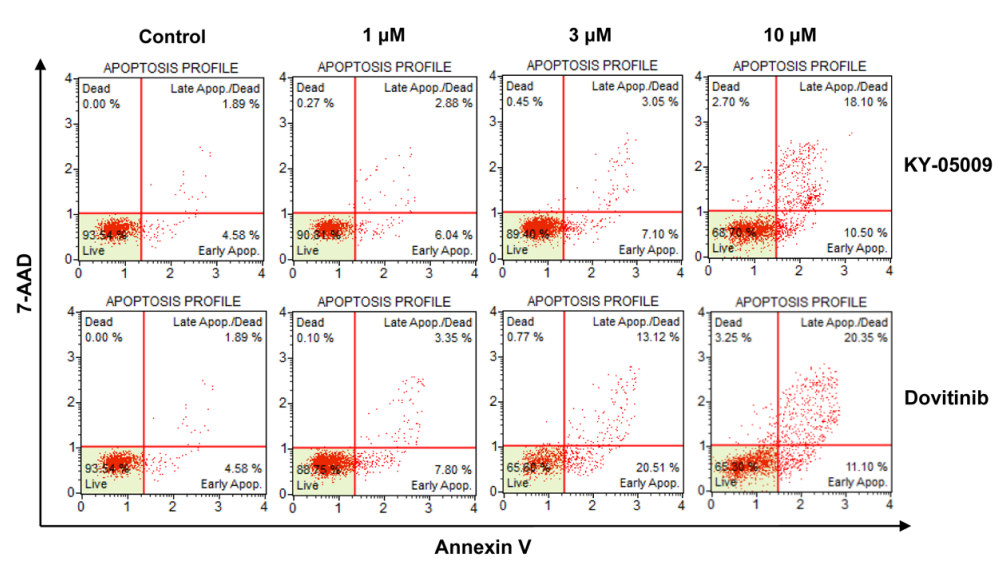

B

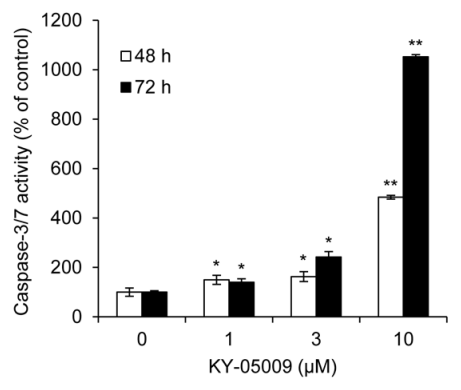

$c$

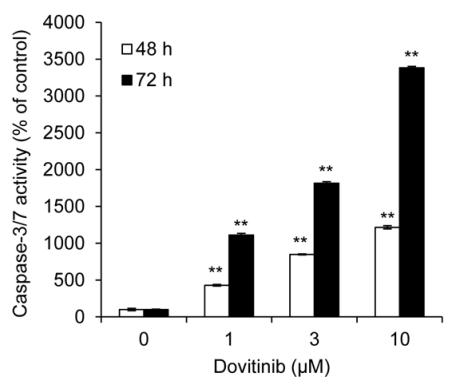

Figure 4: KY-05009 and dovitinib induce apoptosis of MM cells. (A) Flow cytometry results for RPMI8226 cells treated with KY-05009 or dovitinib for $48 \mathrm{~h}$. The apoptosis profile was represented by Annexin V and 7-AAD uptake (Annexin V versus 7-AAD). Each reported result is representative of triplicate experiments. (B and C) RPMI8226 cells were treated with KY-05009 (B) or dovitinib (C) for 48 or $72 \mathrm{~h}$ and the relative caspase- $3 / 7$ activity measured. Data are presented as mean \pm SD. Experiments were performed in triplicate. ${ }^{*} P<$ $0.01,{ }^{* *} P<0.001$ versus control. 
TCF4, but KY-05009 and dovitinib suppressed the transcriptional activity of these genes (Figure 6C).

We confirmed the synergistic effect of KY-05009 and dovitinib through immunoprecipitation. As shown in Figure 6D, IL-6 treatment time of $9 \mathrm{~h}$ increased the phosphorylation of TCF4. The IL-6-induced interaction between TCF4 and $\beta$-catenin and the phosphorylation of TCF4 were inhibited by KY-05009 or dovitinib or combined treatment. Although $9 \mathrm{~h}$ of treatment did not significantly inhibit TNIK expression, TNIK expression and phosphorylation in cytosol and nuclear fractions were inhibited by KY-05009 and dovitinib after $18 \mathrm{~h}$ treatment (Figure 6E). These results indicate that the inhibitory effect of KY-05009 and dovitinib could contribute to the IL-6-induced proliferation of MM cells through the suppression of TNIK-mediated Wnt signaling.

\section{DISCUSSION}

In $\mathrm{MM}$, a canonical $\mathrm{Wnt} / \beta$-catenin signaling pathway is endogenously activated or stimulated with Wnt ligands, increasing MM cell survival [23]. TNIK was introduced as an important transcriptional mediator in the canonical Wnt signaling pathway [6, 7]. In addition, overexpression of nuclear TNIK is related to the prognosis of cancer [9]. These previous studies indicate that TNIK-mediated activation of Wnt signaling could be a therapeutic target in the development of anti-cancer agents.

Silencing of endogenous TNIK and inhibition of TNIK kinase activity suppressed the proliferation of MM cells and induced caspase-dependent apoptosis [11]. Dovitinib also inhibited the expression of TNIK and the interaction with TCF4 in the nucleus. Phosphorylation of TCF4 was suppressed by dovitinib treatment, which then inhibited the transcriptional activity of Wnt target genes. These results provide crucial information that the inhibition of endogenous TNIK induces the suppression of Wnt signaling regulating MM cell survival.

IL-6 has been identified as a major growth factor for the differentiation of B cells to antibody-producing plasma cells and in myeloma cell growth [13-16, 24]. To investigate the stimulation effect of IL- 6 on cell proliferation and TNIK expression in Wnt signaling, we used the RPMI8226 MM cell line, which responds to exogenous IL-6 treatment but does not express IL-6
A

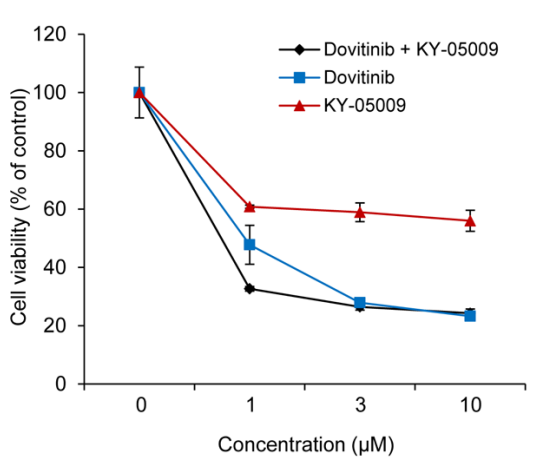

B

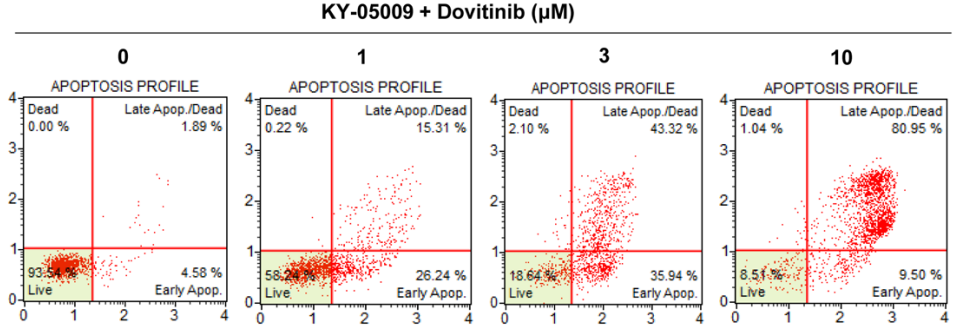

C

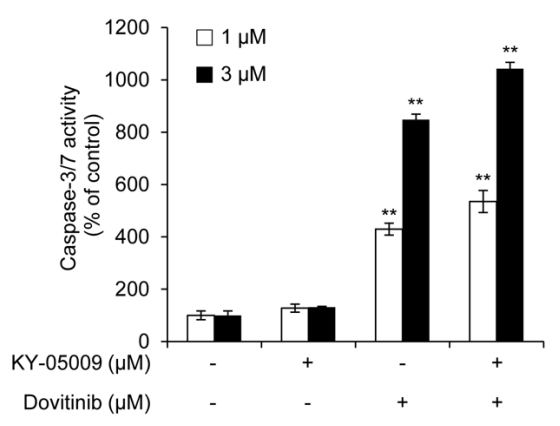

D

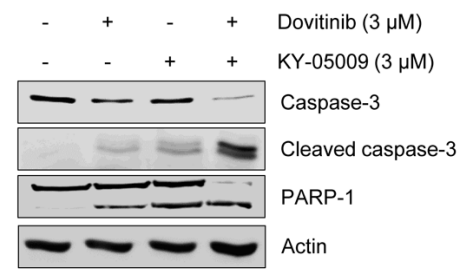

Figure 5: Synergistic effect of KY-05009 and dovitinib induces apoptosis of MM cells. (A) RPMI8226 cells were treated with KY-05009 alone or combination with dovitinib $(0-10 \mu \mathrm{M})$ for $48 \mathrm{~h}$ and cell viability measured. (B) The apoptotic population of KY-05009 and dovitinib co-treated cells was analyzed by flow cytometry. Each reported result is representative of triplicate experiments. (C) RPMI8226 cells were treated with KY-05009 and dovitinib (1 or $3 \mu \mathrm{M})$ for $48 \mathrm{~h}$ and the relative caspase 3/7 activity measured. Data are presented as mean \pm SD. Experiments were performed in triplicate. ${ }^{* *} P<0.001$ versus control. (D) Western blot of cleaved caspase-3 expression in the cytosolic fraction and PARP-1 in the nuclear fraction of RPMI8226 cells treated with KY-05009 or dovitinib alone or in combination for $48 \mathrm{~h}$. Actin was used as a loading control. 
Table 2: Combination index (CI) values for the two-drug combination against RPMI8226 cell viability

\begin{tabular}{cccc}
\hline KY-05009 $(\boldsymbol{\mu M})$ & & Dovitinib $(\boldsymbol{\mu M})$ & CI value \\
\hline 1 & 1 & 0.2210 \\
1 & 3 & 0.3428 \\
1 & 10 & 0.7951 \\
3 & 1 & 0.1971 \\
3 & 3 & 0.3661 \\
3 & 10 & 0.7778 \\
10 & 1 & 0.1939 \\
10 & 3 & 0.2906 \\
10 & 10 & 0.7474 \\
\hline
\end{tabular}

A

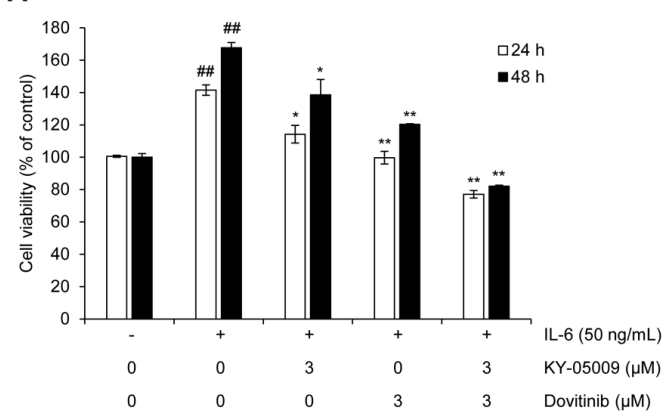

C

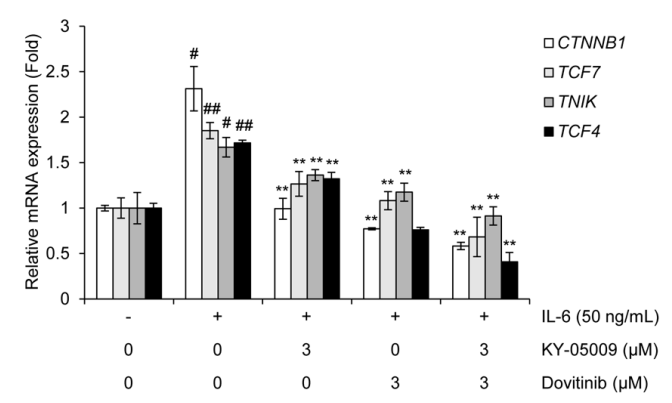

E

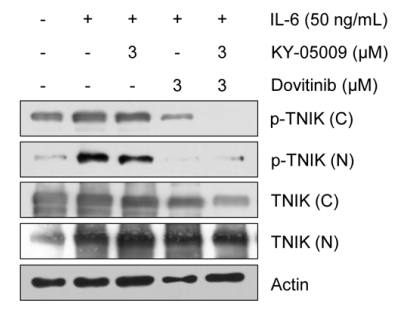

B

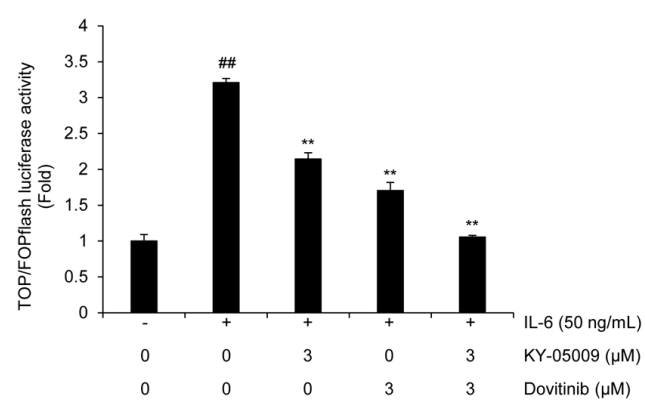

D

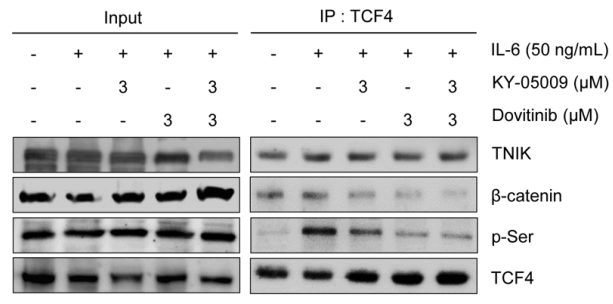

Figure 6: Inhibition of TNIK suppresses IL-6-induced proliferation and the Wnt signaling pathway in MM cells. (A) Cell viability of RPMI8226 cells treated with IL-6 $(50 \mathrm{ng} / \mathrm{mL})$ and KY-05009 $(3 \mu \mathrm{M})$ or dovitinib $(3 \mu \mathrm{M})$ alone or in combination for 24 or $48 \mathrm{~h}$. Data are presented as mean \pm SD. Experiments were performed in triplicate. (B) Relative TCF/LEF luciferase activity measured by FOPflash-normalized TOPflash luciferase activityin RPMI8226 cells treated with IL-6 (50 ng/mL) and KY-05009 (3 $\mu$ M) or dovitinib (3

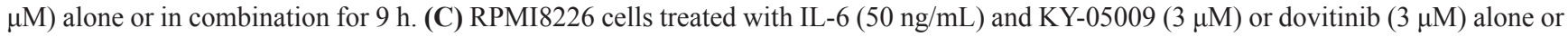
in combination for $1 \mathrm{~h}$. The mRNA expression of indicated genes was detected by qRT-PCR analysis. (D and E) The expression of TCF4interacting proteins and phosphorylation of TNIK detected by immunoprecipitation and Western blot of RPMI8226 cells treated with IL-6 $(50 \mathrm{ng} / \mathrm{mL})$ and KY-05009 $(3 \mu \mathrm{M})$ or dovitinib $(3 \mu \mathrm{M})$ alone or in combination for $9 \mathrm{~h} .{ }^{*} P<0.01,{ }^{* *} P<0.001$ versus control; ${ }^{\#} P<0.01$, ${ }^{\# \#} P$ $<0.001$ versus cells treated with IL- 6 alone. 
or IL-6 receptor transcripts [24]. Based on our recent report, we hypothesized that targeting TNIK and the Wnt signaling pathway may be a novel therapeutic approach in the development of new drugs for MM treatment. Exogenous IL-6 did not significantly activate the production of other cytokines in MM cells, but increased concentrations of IL- 6 dose- and time-dependently stimulated the proliferation of MM cells. Similarly, IL-6 treatment increased the mRNA expression of Wnt target genes and TNIK protein expression. These results suggest that IL-6-induced activation of TNIK-mediated Wnt signaling influences MM cell proliferation.

KY-05009 is a novel aminothiazole developed as a TNIK inhibitor that exhibits anti-cancer activity in human colorectal cancer cells [25]. KY-05009 had high affinity for the ATP binding site of TNIK that plays a crucial role in the inhibition of kinase activity $\left(K_{\mathrm{i}}=100\right.$ $\mathrm{nM}$ ) [12]. In addition, KY-05009 inhibited the interaction between TCF4 and TNIK/ $\beta$-catenin that suppressed the phosphorylation of TCF4. This report provides pivotal information that the inhibition of TNIK and Wnt signaling by KY-05009 can be a new therapeutic strategy for the treatment of Wnt signaling-activated cancer cells. Our study also suggested that inhibition of Wnt signaling by TNIK inhibitors can suppress the IL-6-induced proliferation of MM cells. The anti-cancer activity of KY-05009 was elevated by co-treatment with dovitinib, a selective receptor tyrosine kinase inhibitor. Although dovitinib exerts inhibitory effects on various kinases, it also exhibits high binding affinity for the ATP binding site of TNIK with an inhibiting effect on the kinase activity $[11,26-28]$. We confirmed that dovitinib has an inhibitory effect on the expression of Wnt signaling modulators, such as $\beta$-catenin and TCF4, as well as TNIK, in MM cells [11]. Previous studies support our results that inhibition of TNIK by treatment with both KY-05009 and dovitinib exerts synergistic effect on the proliferation of MM cells.

Recent studies have described the potential of TNIK as an anti-cancer target to inhibit Wnt signaling in several types of human cancers [4, 6-11] and TNIK inhibitors have been developed to treat Wnt-active cancers, but more experimental evidence is needed to elucidate the mechanism underlying the inhibition of proliferation in cancers. Although the biological function of TNIK in hematological cancers, such as MM and leukemia, has not been clearly investigated yet, our results suggest a new approach for treating MM stimulated by exogenous IL6 . Based on our results, we hypothesize that exogenous IL-6, in a paracrine or autocrine manner, elevates the expression of TNIK and the subsequent activation of Wnt signaling, which enhances MM cell survival. In particular, our results show that the combinatorial use of existing kinase inhibitors with an inhibitory effect on TNIK kinase activity and TNIK inhibitors may be an effective therapeutic strategy for the treatment of MM.
As a rare hematological disease, the methods for treating MM are still limited, but increasing data on TNIK and its biological function in human cancers will be crucial for the development of TNIK-targeting drugs.

\section{MATERIALS AND METHODS}

\section{Cell culture and PBMC preparation}

The human MM RPMI8226 cell line was obtained from the Korean Cell Line Bank (\#10155). The use of human primary PBMCs and MM patient's blood samples for this study was approved by the International Review Board of Eulji University (EU 16-10). All cells were maintained in RPMI-1640 medium (Corning Inc., NY, USA) containing $5 \%$ fetal bovine serum (FBS) (Corning Inc., NY, USA) and $1 \%$ antibiotics $(100 \mathrm{U} / \mathrm{mL}$ penicillin and $100 \mu \mathrm{g} / \mathrm{mL}$ streptomycin, HyClone ${ }^{\mathrm{TM}}$, GE Healthcare, Salt Lake City, UT, USA) in a humidified atmosphere of $5 \% \mathrm{CO}_{2}$ at $37^{\circ} \mathrm{C}$.

\section{Cell viability assay}

PBMCs or RPMI8226 cells $\left(1.0 \times 10^{4}\right.$ cells/well $)$ were seeded in 96-well plates and incubated for $24 \mathrm{~h}$. After incubation, the cells were treated with dovitinib (Selleck Chemicals, USA) and/or KY-05009 [12] in complete medium containing 5\% FBS for $24-72 \mathrm{~h}$. Cell viability was measured using the Cell Counting Kit-8 (Dojindo Molecular Technologies, Kumamoto, Japan) according to the manufacturer's instructions. The absorbance was measured using the Multiscan ${ }^{\mathrm{TM}}$ FC microplate photometer (Thermo Fisher Scientific, Boston, MA, USA). Experiments were performed in triplicate.

\section{Human cytokine array}

The cytokine levels after stimulation with human IL-6 were measured using the Proteome Profiler ${ }^{\mathrm{TM}}$ Cytokine Array Kit (R\&D Systems, MN, USA). RPMI8226 cells were seeded in $60-\mathrm{mm}^{2}$ dishes in culture medium containing 5\% FBS for $24 \mathrm{~h}$. The cells were replenished with serum-free medium for $24 \mathrm{~h}$ before treatment with recombinant human IL-6 (10 ng/mL) (R\&D Systems, MN, USA) for $18 \mathrm{~h}$. After incubation, all experimental procedures were completed according to the manufacturer's instructions.

\section{Flow cytometry}

RPMI8226 cells $\left(1 \times 10^{5}\right.$ cells $\left./ \mathrm{mL}\right)$ were seeded in 6-well plates and treated with KY-05009 and/or dovitinib $(0-10 \mu \mathrm{M})$ in complete medium containing 5\% FBS for 48 h. Harvested cells were washed with phosphate-buffered saline (PBS) and treated using the MUSE ${ }^{\mathrm{TM}}$ Annexin V Dead Cell Assay Kit (Merck Millipore, Darmstadt, 
Germany). The population of apoptotic and dead cells was determined using the MUSETM Cell Analyzer (Merck Millipore, Darmstadt, Germany) and acquired data analyzed using the MUSETM Annexin V and Dead Cell software module (Merck Millipore, Darmstadt, Germany) according to the manufacturer's instructions.

\section{Western blot analysis}

Cytoplasmic and nuclear fractions were prepared from RPMI8226 cell lysates using NE-PER nuclear and cytoplasmic extraction reagent (Thermo Fisher Scientific, Boston, MA, USA). After protein quantification, cytoplasmic or nuclear proteins were loaded on polyacrylamide gels for electrophoresis and the separated proteins transferred to nitrocellulose membranes. Protein expression was detected using specific primary antibodies against p-TNIK, TNIK, TCF4, Lamin B1, $\beta$-catenin, and actin (Santa Cruz Biotechnology, Inc., Austin, TX, USA); PARP-1, caspase-3, and cleaved caspase-3 (Cell Signaling Technology, Inc., Danvers, MA, USA); and phosphoserine (p-Ser; Abcam, Cambridge, UK). After incubation with horseradish peroxidase (HRP)-conjugated secondary antibodies, the signals were visualized using Luminata ${ }^{\mathrm{TM}}$ Forte Western HRP Substrate (Merck Millipore, Darmstadt, Germany). The band intensities were measured to determine the relative protein expression using X-ray films and development solution (Fujifilm, Tokyo, Japan). Bands were quantified using ImageJ software. Actin was used as a loading control.

\section{Immunoprecipitation}

Total lysates of RPMI8226 cells were pre-cleared with normal control immunoglobulin $G$ and Protein A agarose beads (Thermo Fisher Scientific, Boston, MA, USA) after protein quantification. Endogenous protein complexes were immunoprecipitated overnight at $4^{\circ} \mathrm{C}$ with an anti-TCF4 antibody (Santa Cruz Biotechnology, Inc., Austin, TX, USA) plus Protein A agarose beads. TCF4bound proteins were subjected to $4 \%-20 \%$ polyacrylamide gel electrophoresis and Western blot performed. TCF4bound proteins were detected using specific primary antibodies against TNIK, p-Ser, and $\beta$-catenin. After incubation with HRP-conjugated secondary antibodies, the signals were visualized using Luminata ${ }^{\mathrm{TM}}$ Forte Western HRP Substrate (Merck Millipore, Darmstadt, Germany). The band intensities were measured to determine the relative protein expression using X-ray films and development solution (Fujifilm, Tokyo, Japan).

\section{TOP/FOPflash reporter luciferase assay}

RPMI8226 cells were trasfected with TOPflash TCF reporter plasmid (wild-type TCF binding site) (Merck Millipore, Darmstadt, Germany), FOPflash plasmid (mutant TCF binding site) (Merck Millipore,
Darmstadt, Germany), and Lipofectamine ${ }^{2000}$ (Thermo Fisher Scientific, Boston, MA, USA) in an antibioticfree medium. FOPflash-normalized TOPflash luciferase activity was represented as the relative TCF/LEF luciferase activity. After TOP/FOPflash reporter transfection, serumdeprived cells were treated with IL-6 (50 ng/mL) and KY05009, dovitinib, or both for $48 \mathrm{~h}$. Cells were washed with PBS, and then lysed using passive lysis buffer (Promega, Madison, WI, USA). The luciferase activity was evaluated using the luciferase reporter assay (Promega, Madison, WI, USA).

\section{Measurement of caspase-3/7 activity}

RPMI8226 cells $\left(2 \times 10^{5}\right.$ cells $\left./ \mathrm{mL}\right)$ were treated with dovitinib and/or KY-05009 (1 or $3 \mu \mathrm{M})$ in RPMI1640 medium containing 5\% FBS for $48 \mathrm{~h}$. After cell harvesting, caspase-3/7 activity was measured using Caspase-Glo ${ }^{\circledR}$ 3/7 Assay Systems (Promega, Madison, WI, USA). Detailed experiments were performed as described in the manufacturer's instructions.

\section{Quantitative real-time RT-PCR (qRT-PCR)}

Total RNA was isolated from the frozen buffy coat specimens of human MM patients using the NucleoSpin ${ }^{\circledR}$ RNA Blood extraction kit (Macherey-Nagel, GmbH\&Co. KG, Germany). RPMI8226 cells were treated with IL-6 (10 ng/mL) in RPMI-1640 medium containing 0.1\% FBS for 0 - $60 \mathrm{~min}$. After incubation, the total RNA was isolated from RPMI8226 cells using the AccuPrep ${ }^{\circledR}$ RNA Extraction Kit (Bioneer Corp., Daejeon, Korea) and the cDNA synthesized from $1 \mu \mathrm{g}$ of total RNA using oligo (dT) primers (Bioneer Corp., Daejeon, Korea) and the RocketScript $^{\mathrm{TM}}$ Reverse Transcriptase Kit (Bioneer Corp., Daejeon, Korea). Quantitative real-time RT-PCR was performed using ExcelTaq 2X Q-PCR Master Mix (SMOBiO, Hsinchu, Taiwan) and the CFX96 ${ }^{\mathrm{TM}}$ RealTime PCR System (Bio-Rad, Sacramento, CA, USA). The cycling conditions were as follows: $95^{\circ} \mathrm{C}$ for $3 \mathrm{~min}$ followed by 40 cycles at $95^{\circ} \mathrm{C}$ for $15 \mathrm{~s}, 60^{\circ} \mathrm{C}$ for $30 \mathrm{~s}$, and $72^{\circ} \mathrm{C}$ for $30 \mathrm{~s}$. Table 1 lists the target-specific primer sequences. All reactions were performed in triplicate with GAPDH as an internal standard. The data were analyzed using the $2_{T}^{-\Delta \Delta C}$ method [29].

\section{Analysis of combined drug effects}

The effect of the combined drugs was analyzed using the Calcusyn software program (Biosoft, Cambridge, UK) and CI methods derived from the median effect principle of Chou and Talalay [30] to determine whether the combined treatment of two compounds was additive or synergistic. The CI was calculated by the formula [31]; a CI of 1 indicated an additive effect between the two compounds, whereas a CI $>1$ or $\mathrm{CI}<1$ indicated antagonism or synergism, respectively. 


\section{Statistical analysis}

All data are reported as mean \pm SD. Significant differences were determined by the Student's $t$-test.

\section{ACKNOWLEDGMENTS}

This work was supported by the Basic Science Research Program through the National Research Foundation of Korea (NRF) grant funded by the Ministry of Science, ICT, and Future Planning (NRF2014R1A1A1002349). The biospecimens for this study were provided by the Chonbuk University Hospital Biobank, a member of the Korea Biobank Network, which is supported by the Ministry of Health, Welfare, and Family Affairs, Republic of Korea. All samples derived from the National Biobank of Korea were obtained with informed consent under institutional review boardapproved protocols (T16-7).

\section{CONFLICTS OF INTEREST} interest.

All authors declare that they have no conflicts of

\section{REFERENCES}

1. Kyle RA, Rajkumar SV. Multiple myeloma. Blood. 2008; 111: 2962-72.

2. Ria R, Reale A, Vacca A. Novel agents and new therapeutic approaches for treatment of multiple myeloma. World J Methodol. 2014; 4: 73-90.

3. Rajkumar SV, Kyle RA. Multiple myeloma: diagnosis and treatment. Mayo Clin Proc. 2005; 80: 1371-82.

4. Fu CA, Shen M, Huang BC, Lasaga J, Payan DG, Luo Y. TNIK, a novel member of the germinal center kinase family that activates the c-Jun N-terminal kinase pathway and regulates the cytoskeleton. J Biol Chem. 1999; 274: 30729-37.

5. Taira K, Umikawa M, Takei K, Myagmar BE, Shinzato M, Machida N, Uezato H, Nonaka S, Kariya K. The Traf2and Nck-interacting kinase as a putative effector of Rap2 to regulate actin cytoskeleton. J Biol Chem. 2004; 279: 49488-96.

6. Mahmoudi T, Li VS, Ng SS, Taouatas N, Vries RG, Mohammed S, Heck AJ, Clevers H. The kinase TNIK is an essential activator of Wnt target genes. EMBO J. 2009; 28: 3329-40.

7. Shitashige M, Satow R, Jigami T, Aoki K, Honda K, Shibata T, Ono M, Hirohashi S, Yamada T. Traf2- and Nck-interacting kinase is essential for Wnt signaling and colorectal cancer growth. Cancer Res. 2010; 70: 5024-33.
8. Yu DH, Zhang X, Wang H, Zhang L, Chen H, Hu M, Dong Z, Zhu G, Qian Z, Fan J, Su X, Xu Y, Zheng L, et al. The essential role of TNIK gene amplification in gastric cancer growth. Oncogenesis. 2014; 17: e93.

9. Jin J, Jung HY, Wang Y, Xie J, Yeom YI, Jang JJ, Lee KB. Nuclear expression of phosphorylated TRAF2- and NCK-interacting kinase in hepatocellular carcinoma is associated with poor prognosis. Pathol Res Pract. 2014; 210: 621-7.

10. Schürch C, Riether C, Matter MS, Tzankov A, Ochsenbein AF. CD27 signaling on chronic myelogenous leukemia stem cells activates Wnt target genes and promotes disease progression. J Clin Invest. 2012; 122: 624-38.

11. Chon HJ, Lee Y, Bae KJ, Byun BJ, Kim SA, Kim J. Traf2and Nck-interacting kinase (TNIK) is involved in the anticancer mechanism of dovitinib in human multiple myeloma IM-9 cells. Amino Acids. 2016; 48: 1591-9.

12. Kim J, Moon SH, Kim BT, Chae CH, Lee JY, Kim SH. A novel aminothiazole KY-05009 with potential to inhibit Traf2- and Nck-interacting kinase (TNIK) attenuates TGF- $\beta 1$-mediated epithelial-to-mesenchymal transition in human lung adenocarcinoma A549 cells. PLoS One. 2014; 9: e110180.

13. Klein B, Zhang XG, Jourdan M, Content J, Houssiau F, Aarden L, Piechaczyk M, Bataille R. Paracrine rather than autocrine regulation of myeloma-cell growth and differentiation by interleukin-6. Blood. 1989; 73: 517-26.

14. Zhang XG, Klein B, Bataille R. Interleukin- 6 is a potent myeloma-cell growth factor in patients with aggressive multiple myeloma. Blood. 1989; 74: 11-3.

15. Anderson $\mathrm{KC}$, Jones RM, Morimoto $\mathrm{C}$, Leavitt $\mathrm{P}$, Barut BA. Response patterns of purified myeloma cells to hematopoietic growth factors. Blood. 1989; 73: 1915-24.

16. Klein B, Zhang XG, Jourdan M, Boiron JM, Portier M, Lu $\mathrm{ZY}$, Wijdenes J, Brochier J, Bataille R. Interleukin-6 is the central tumor growth factor in vitro and in vivo in multiple myeloma. Eur Cytokine Netw. 1990; 1: 193-201.

17. Derksen PW, Tjin E, Meijer HP, Klok MD, MacGillavry HD, van Oers MH, Lokhorst HM, Bloem AC, Clevers H, Nusse R, van der Neut R, Spaargaren M, Pals ST. Illegitimate WNT signaling promotes proliferation of multiple myeloma cells. Proc Natl Acad Sci U S A. 2004; 101: 6122-7.

18. Qiang YW, Endo Y, Rubin JS, Rudikoff S. Wnt signaling in B-cell neoplasia. Oncogene. 2003; 22: 1536-45.

19. Sukhdeo K, Mani M, Zhang Y, Dutta J, Yasui H, Rooney MD, Carrasco DE, Zheng M, He H, Tai YT, Mitsiades C, Anderson KC, Carrasco DR. Targeting the betacatenin/TCF transcriptional complex in the treatment of multiple myeloma. Proc Natl Acad Sci U S A. 2007; 104: 7516-21.

20. Schmid I, Krall WJ, Uittenbogaart CH, Braun J, Giorgi JV. Dead cell discrimination with 7 -amino-actinomycin D in 
combination with dual color immunofluorescence in single laser flow cytometry. Cytometry. 1992; 13: 204-8.

21. Majno G, Joris I. Apoptosis, oncosis, and necrosis: An overview of cell death. Am J Pathol. 1995; 146: 3-15.

22. Vermes I, Haanen C, Steffens-Nakken H, Reutelingsperger C. A novel assay for apoptosis. Flow cytometric detection of phosphatidylserine expression on early apoptotic cells using fluorescein labelled Annexin V. J Immunol Meth. 1995; 184: 39-51.

23. Bommert K, Bargou RC, Stühmer T. Signalling and survival pathways in multiple myeloma. Eur J Cancer. 2006; 42: 1574-80.

24. Barut BA, Zon LI, Cochran MK, Paul SR, Chauhan D, Mohrbacher A, Fingeroth J, Anderson KC. Role of interleukin 6 in the growth of myeloma-derived cell lines. Leuk Res. 1992; 16: 951-9.

25. Yamada T, Shitashige M, Yokota K, Sawa M, Morlyama H. TNIK inhibitor and its use. Google Patents. 2010; US20100216795 A1.

26. Davis MI, Hunt JP, Herrgard S, Ciceri P, Wodicka LM, Pallares G, Hocker M, Treiber DK, Zarrinkar PP. Comprehensive analysis of kinase inhibitor selectivity. Nat Biotechnol. 2011; 29: 1046-51.
27. Lopes de Menezes DE, Peng J, Garrett EN, Louie SG, Lee SH, Wiesmann M, Tang Y, Shephard L, Goldbeck C, Oei Y, Ye H, Aukerman SL, Heise C. CHIR-258: a potent inhibitor of FLT3 kinase in experimental tumor xenograft models of human acute myelogenous leukemia. Clin Cancer Res. 2005; 11: 5281-91.

28. Porta C, Giglione P, Liguigli W, Paglino C. Dovitinib (CHIR258, TKI258): structure, development and preclinical and clinical activity. Future Oncol. 2015; 11: 39-50.

29. Livak KJ, Schmittgen TD. Analysis of relative gene expression data using real-time quantitative PCR and the 2(-Delta Delta C(T)). Method Methods. 2001; 25: 402-8.

30. Chou TC, Talalay P. Quantitative analysis of dose-effect relatioships: the combined effects of multiple drugs or enzyme inhibitors. Adv Enzyme Regul. 1984; 22: 27-55.

31. Zhao L, Wientjes MG, Au JL. Evaluation of combination chemotherapy: integration of nonlinear regression, curve shift, isobologram, and combination index analyses. Clin Cancer Res. 2004; 10: 7994-8004. 